\title{
Nonconvulsive Status With Metronidazole
}

The Neurohospitalist

3(4) $185-189$

(C) The Author(s) 2013

Reprints and permission:

sagepub.com/journalsPermissions.nav

DOI: 10.1 | 177/194|8744|2470667

nhos.sagepub.com

(S)AGE

\author{
A. A. Cantador, MD', J. F. Meschia, MD², W. D. Freeman, $M D^{2}$, and \\ W. O. Tatum, DO ${ }^{2}$
}

\begin{abstract}
Objective: To expand the adverse events associated with metronidazole to include nonconvulsive status epilepticus (NCSE). Design: Observational single case report of a rare association. Setting: Hospitalized lung transplant recipient treated with metronidazole for prevention of infection. Patient: A 56-year-old man with systemic symptoms, peripheral neuropathy, generalized seizure, and a subsequent acute deterioration of mental status due to NCSE. Interventions: Administration of midazolam was successful in terminating status epilepticus. Main Outcome Measures: Abrupt termination of NCSE was evident on continuous bedside electroencephalogam associated with clinical resolution of mental status. Results: Recovery occurred from NCSE eventually deteriorating to a fatal outcome. Conclusions: Metronidazole may be associated with successfully treated NCSE.
\end{abstract}

\section{Keywords}

nonconvulsive status epilepticus, EEG, metronidazole, encephalopathy, organ transplant

\section{Introduction}

Metronidazole is a synthetic nitroimidazole antimicrobial that, despite 45 years of extensive use, remains the criterion standard for the management and prophylaxis of anaerobic infections as well as an agent of choice in the treatment of susceptible protozoan infections. ${ }^{1}$ The most serious adverse effects reported with metronidazole include encephalopathy, aseptic meningitis, optic and peripheral neuropathy, and convulsive seizures. ${ }^{2}$ Neuroimaging abnormalities help identify patients with metronidazole encephalopathy (ME), revealing abnormalities that characteristically involve the dentate nuclei of the cerebellum. ${ }^{3}$ Metronidazole-associated seizures have also been reported, ${ }^{4}$ but the spectrum of neurophysiologic complications has not been fully characterized.

We report a recipient of a lung transplant for pulmonary fibrosis with $\mathrm{ME}$ who experienced nonconvulsive status epilepticus (NCSE) that was successfully treated with antiepileptic drugs (AEDs). We expand the complications of ME to include NCSE in patients treated with metronidazole. A higher index of clinical suspicion and early use of electroencephalogram (EEG) may help lead to successful treatment in patients suspected of treated ME.

\section{Case Presentation}

A 56-year-old right-handed man with hypertension, coronary artery disease, and steroid-induced diabetes mellitus underwent a right lung transplant due to idiopathic pulmonary fibrosis. Two months later, he was hospitalized for fever, cough, dyspnea on exertion, severe exercise intolerance, weakness, and postural dizziness associated with a right pleural effusion. His neurological examination demonstrated no abnormal focal or lateralized features. However, generalized weakness persisted and an electromyography demonstrated a mild diffuse sensory-motor peripheral neuropathy. Five weeks later, he had a generalized seizure, which was attributed to the use of immunosuppressant drugs. He was not administered AEDs after a magnetic resonance imaging (MRI) disclosed that there were no structural lesions. Additionally, an EEG demonstrated a diffuse slowing of the background to 7 to $8 \mathrm{~Hz}$ with intermittent typical triphasic waves. A daily dose of $500 \mathrm{mg}$ of intravenous (IV) metronidazole was prescribed to prevent Clostridium difficile infection. Ten days later, myoclonic jerks and an abrupt deterioration in mental status prompted reintubation. An EEG (Figure 1A) revealed continuous generalized periodic epileptiform discharges (GPEDs), suggesting NCSE. Two $7 \mathrm{mg}$ boluses of IV midazolam were administered during continuous EEG (cEEG) monitoring. Following the second bolus, there was immediate cessation of the GPEDs without recurrence. The

\footnotetext{
' Santa Casa de Misericórdia de Itatiba, Itatiba, Brazil

${ }^{2}$ Department of Neurology, Mayo Clinic, Jacksonville, FL, USA
}

Corresponding Author:

William O. Tatum, Department of Neurology, Mayo Clinic, 4500 San Pablo Road, Jacksonville, FL 32224, USA.

Email: Tatum.william@mayo.edu 


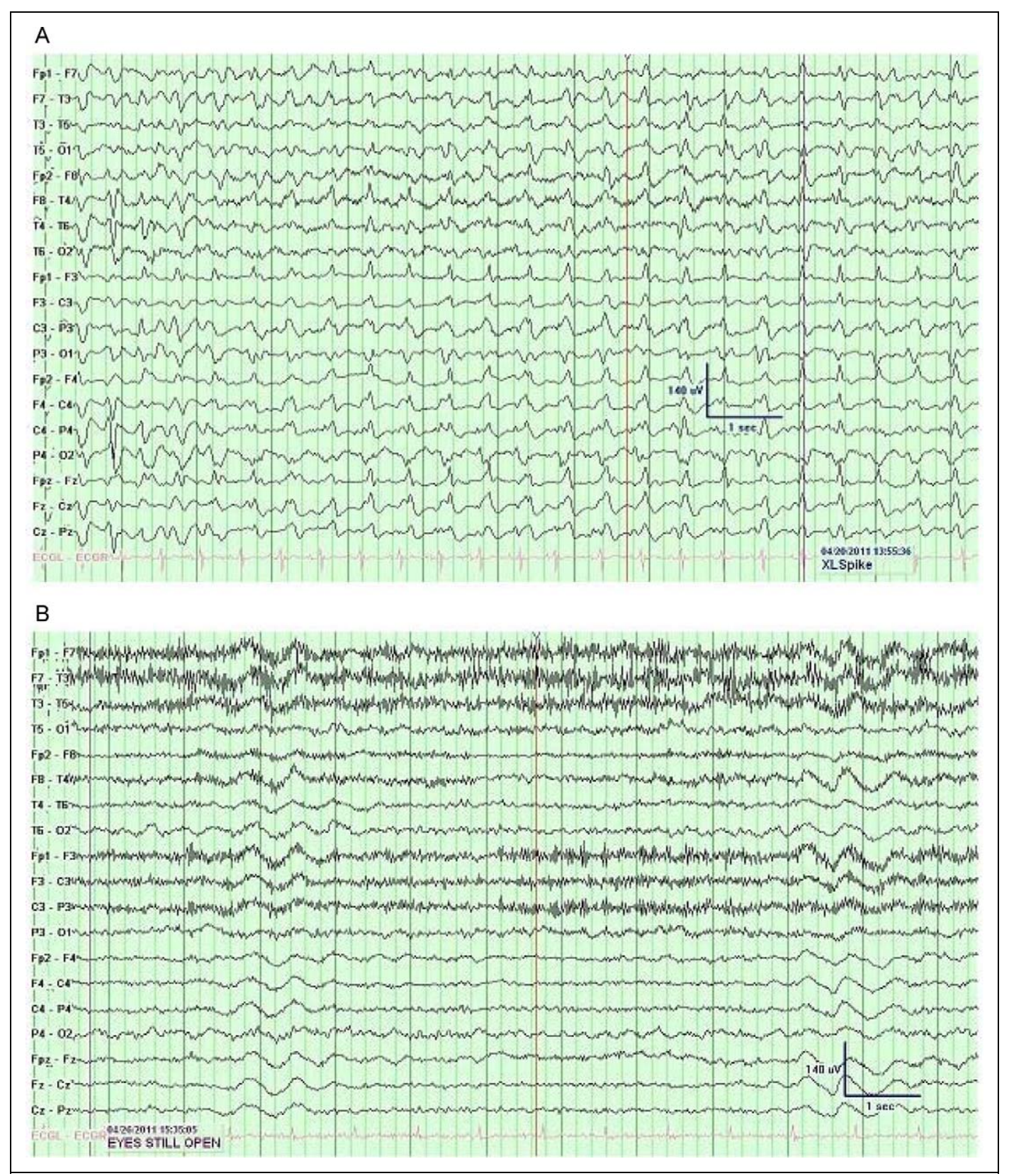

Figure I. A, Electroencephalogram (EEG) demonstrating the generalized periodic epileptiform discharges (GPEDs) of nonconvulsive status epilepticus (NCSE) prior to treatment. B, The EEG immediately following intravenous midazolam demonstrating elimination of GPEDs with improvement in the electrocerebral activity.

background activity also returned to normal with intermixed 4 to $6 \mathrm{~Hz} \theta$ and frequent 1 - to 4 -second bursts of frontal intermittent rhythmic delta activity (Figure 1B). A repeat MRI of the brain obtained 49 days after the initial nondiagnostic study revealed the characteristic features of ME demonstrating abnormal symmetric bilateral fluid attenuated inversion recovery and $\mathrm{T} 2$ hyperintensities involving the dentate nuclei (Figure 2), posterior pons and mid-brain (Figure 3), portions of the superior and middle cerebellar peduncles, and corpus callosum (Figure 4). Hypertrophic olivary degeneration was also present with an appearance and distribution consistent with ME (Figure 5). Metronidazole was then discontinued. A follow-up MRI of the brain was obtained 17 days later, though no significant change or sign of reversibility was evident. The patient initially demonstrated clinical improvement and regained an ability to communicate. He ultimately deteriorated succumbing to overwhelming sepsis 2 months later.

\section{Discussion}

Infection is one of the leading causes of morbidity and mortality after solid organ transplantation. This is due to the potent 


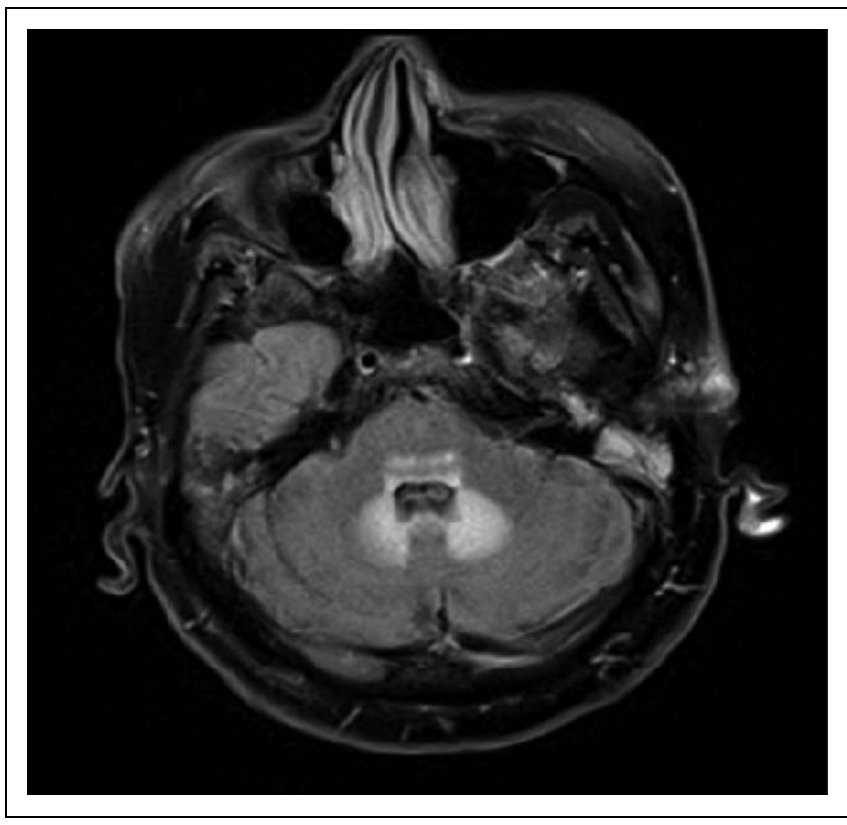

Figure 2. Hyperintense lesion of the dentate nucleus and middle cerebellar peduncles in the fluid attenuated inversion recovery (FLAIR) sequences noted on transverse sections of brain magnetic resonance imaging $(M R I)$.

immunosuppressive treatments required to reduce the rate of acute rejection, which places the patients at a high risk of bacterial, fungal, and viral infections. ${ }^{5}$ Organ transplantation is a growing discipline that frequently utilizes metronidazole to prevent anaerobic infections in high-risk or immunologically compromised hosts. ${ }^{5}$ Our patient with lung transplant experienced an acute decline in mental status associated with ME that followed IV metronidazole use with the associated characteristic findings on MRI of the brain. Although the inferior olivary complex may be affected in patients with $\mathrm{ME}$ and involve the triangle of Guillain and Mollaret, ${ }^{6}$ our patient did not demonstrate palatal myoclonus. The EEG demonstrated GPEDs and clinically, treatment for NCSE resulted in improvement in the patient's mental status with dramatic resolution of NCSE on EEG immediately following IV midazolam. The typical MRI findings in ME demonstrate T2 hyperintense lesions on T2-weighted and fluid-attenuated inversion recovery sequences in the cerebellar dentate nuclei $^{3}$ demonstrated in Figure 2. Kim et al have described a distinctive lesion distribution involving bilateral symmetric T2 hyperintense lesions not only in the cerebellar dentate nucleus but also in the mid-brain dorsal pontine region, medulla, and the corpus callosum. ${ }^{7}$ These findings were present in our patient (Figures 2-5). Other leukoencephalopathies such as posterior reversible encephalopathy syndrome may occur in patients who underwent transplant and also be associated with $\mathrm{NCSE},{ }^{8}$ though the lesion locations on MRI and clinical course help distinguish these conditions. The mechanism of ME is unknown, yet the features on brain
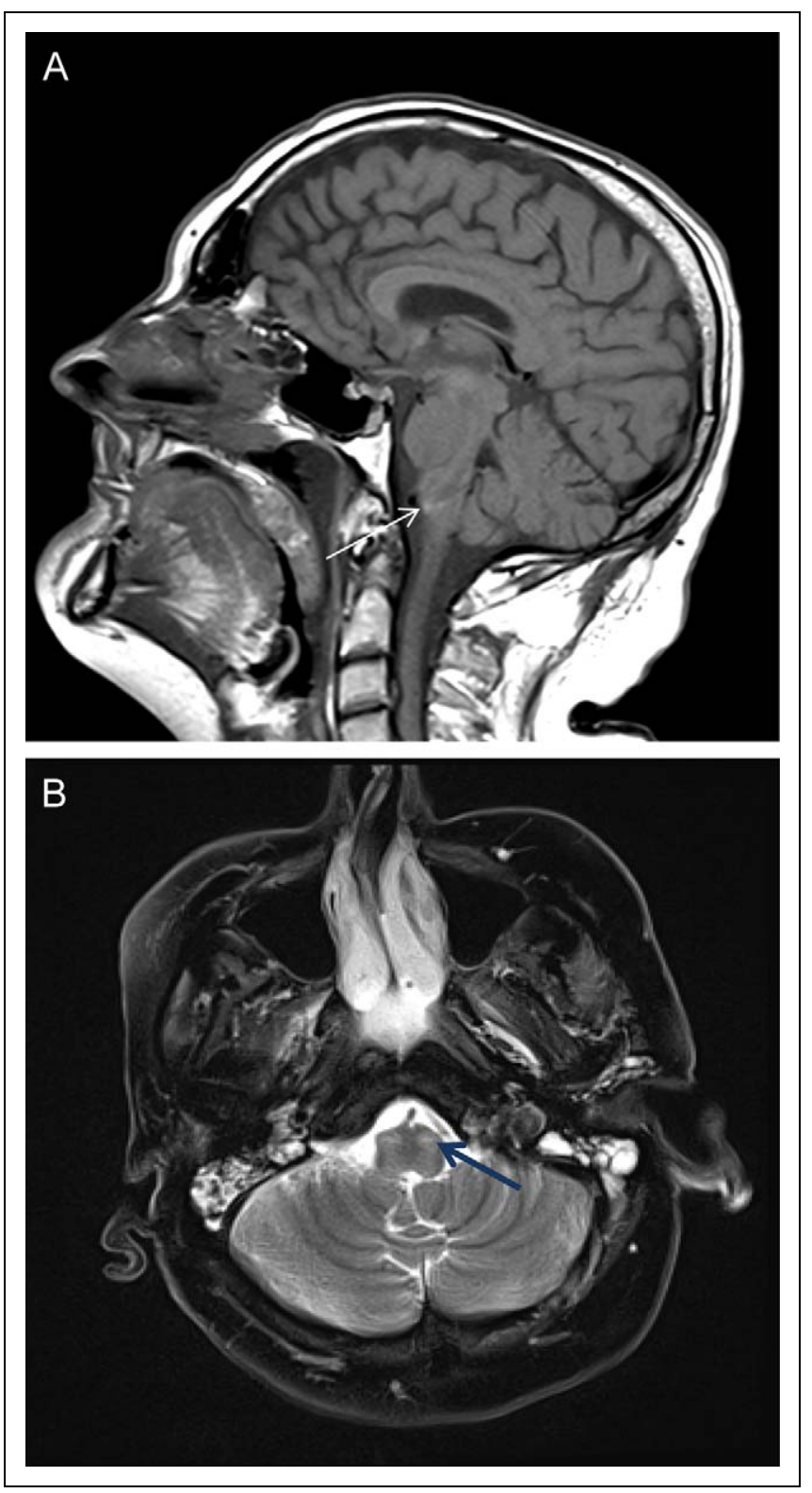

Figure 3. Hyperintense lesion on sagittal TI images (A) and transverse $\mathrm{T} 2$ images (B) demonstrating hypertrophic olivary nuclei on brain magnetic resonance imaging (MRI).

MRI may be reversible potentially reflecting the presence of interstitial edema. ${ }^{9}$ The temporal course has been described by Goyal et al in a series of 45 patients with increased signal changes noted on T2-weighted images involving the inferior olivary nuclei in 1 month following the inciting event. ${ }^{6}$ It may also occur despite normal serum levels ${ }^{9}$ or in shorter and lower dose exposures as in our patient. Patients with liver disease may be predisposed, though our lung transplant patient may have possessed similar issues of immunocompetency, making him more susceptible.

The cumulative dose and extended duration of metronidazole use have been associated with seizures. ${ }^{2,4}$ In patients with encephalopathy, generalized periodic discharges may be 

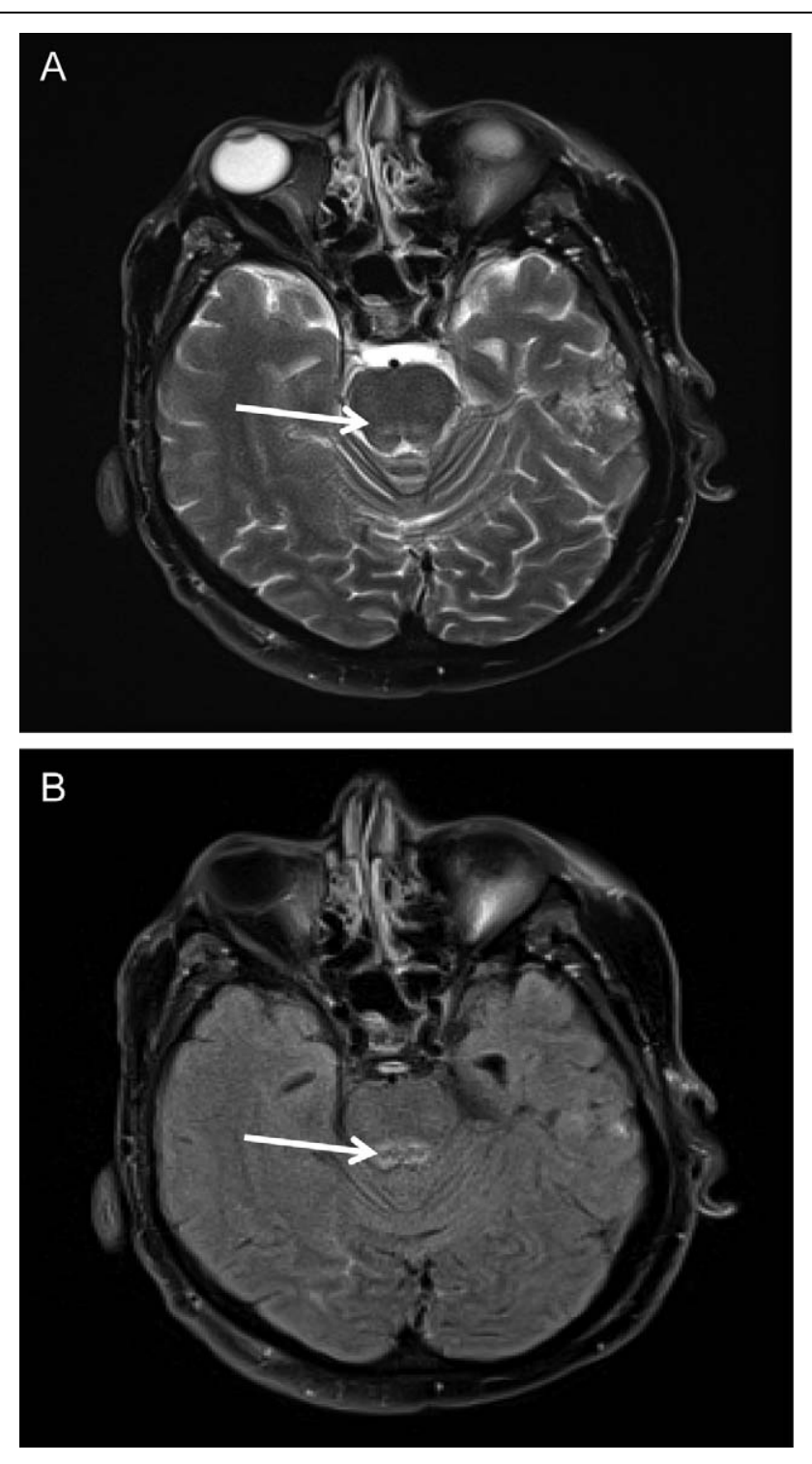

Figure 4. Hyperintense lesion on transverse $T 2$ images $(A)$ and transverse fluid attenuated inversion recovery (FLAIR) images (B) on brain magnetic resonance imaging (MRI).

difficult to distinguish from triphasic waves associated with metabolic encephalopathies and, when nearly continuous, are difficult to separate from NCSE. The finding of clinical improvement with electrographic resolutions of GPEDs on EEG with AEDs or benzodiazepines helps identify NCSE in patients, as in our case. ${ }^{10}$ Electrographic improvement is frequently observed before clinical improvement, making the diagnosis of NCSE challenging, especially when a multifactorial encephalopathy is present in patients in ICU. Furthermore, triphasic waves associated with metabolic encephalopathies can mimic GPEDs and may be reduced with benzodiazepines, thereby creating diagnostic difficulty. ${ }^{11}$ The GPEDs in our patient were most likely the result of NCSE with immediate electroclinical improvement and a convulsion

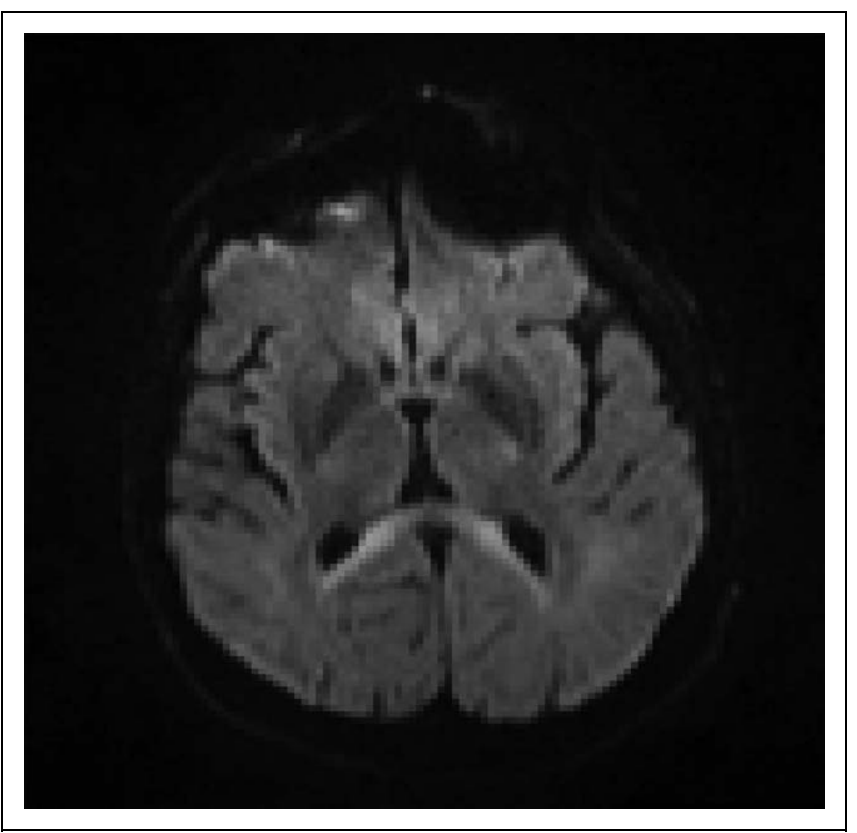

Figure 5. Hyperintense lesion on brain magnetic resonance imaging (MRI) obtained with transverse fluid attenuated inversion recovery (FLAIR) images of the corpus callosum.

that was clinically evident. The combined proconvulsant effects of the medications, which included immunosuppressant drugs and a compromised state, likely played a multifactorial role in developing NCSE in our patient with ME. The fact that the GPEDs noted on the EEG were totally eliminated and did not recur with both an improved background electrocerebral activity and a clinical state support a diagnosis of successfully treated NCSE.

Single case reports limit the strength of an association though unique characteristics may not be readily reproducible or recognized without them. ${ }^{9}$ A recent systematic review demonstrated approximately $15 \%$ of the patients with ME manifest seizures. ${ }^{12}$ We suggest that NCSE may also occur in patients with ME. The EEG should be performed early when encephalopathic signs are first evident and cEEG monitoring pursued when NCSE is encountered.

We conclude that NCSE may occur in patients with ME who undergo solid organ transplantation. Combined metronidazole and immunosuppressant therapy may predispose patients with lung transplant to nonconvulsive seizures in ME. Discontinuation of metronidazole is usually sufficient for improvement in clinical and structural findings in most patients. Nevertheless, emergency treatment with AEDs may be required leading to successful seizure termination and this association should be considered in patients with ME and an abrupt or prolonged encephalopathy.

\section{Declaration of Conflicting Interests}

The authors declared no potential conflicts of interest with respect to the research, authorship, and/or publication of this article. 


\section{Funding}

The authors received no financial support for the research, authorship, and/or publication of this article.

\section{References}

1. Lofmark S, Edlund C, Nord CE. Metronidazole is still the drug of choice for treatment of anaerobic infections. Clin Infect Dis. 2010;50(supp1 1):S16-S23.

2. Finegold SM. Metronidazole. Ann Intern Med. 1980;93(4): 585-587.

3. Woodruff BK, Wijdicks EF, Marshall WF. Reversible metronidazole-induced lesions of the cerebellar dentate nuclei. N Engl J Med. 2002;346(1):68-69.

4. El-Bitar MK, Boustany RM. Common causes of uncommon seizures. Pediatr Neurol. 2009;41(2):83-87.

5. Treede H, Reichenspurner H, Meiser BM, et al. Incidence and spectrum of infections in lung transplanted patients: comparison of four different immunosuppressive protocols. Transplant Proc. 2001;33(1-2):1620-1621.

6. Goyal M, Versnick E, Tuite P, et al. Hypertrophic olivary degeneration: metaanalysis of the temporal evolution of
MR findings. AJNR Am J Neuroradiol. 2000;21(6): 1073-1077.

7. Kim E, Na DG, Kim EY, Kim JH, Son KR, Chang KH. MR imaging of metronidazole-induced encephalopathy: lesion distribution and diffusion-weighted imaging findings. Am J Neuroradiol. 2007;28(9):1652-1658.

8. Palma JA, Gomez-Ibanez A, Martin B, Urrestarazu E, Gil-Bazo I, Pastor MA. Nonconvulsive status epilepticus related to posterior reversible leukoencephalopathy syndrome induced by cetuximab. Neurologist. 2011;17(5):273-275.

9. Ahmed A, Loes DJ, Bressler EL. Reversible magnetic resonance imaging findings in metronidazole-induced encephalopathy. Neurology. 1995;45(3 pt 1):588-589.

10. Husain AM, Mebust KA, Radtke RA. Generalized periodic epileptiform discharges: etiologies, relationship to status epilepticus, and prognosis. J Clin Neurophysiol. 1999;16(1):51-58.

11. Fountain NB, Waldman WA. Effects of benzodiazepines on triphasic waves: implications for nonconvulsive status epilepticus. J Clin Neurophysiol. 2001;18(4):345-352.

12. Kuriyama A, Jackson JL, Doi A, Kamiya T. Metronidazoleinduced central nervous system toxicity: a systematic review. Clin Neuropharmacol. 2011;34(6):241-247. 